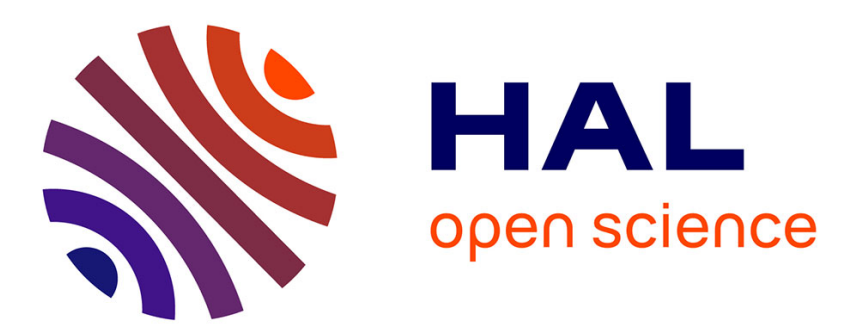

\title{
New analytical method to evaluate the powerplant and chassis coupling in the improvement vehicle $\mathrm{NVH}$
}

Eric Courteille, Lionel Leotoing, Frédéric Mortier, Eric Ragneau

\section{To cite this version:}

Eric Courteille, Lionel Leotoing, Frédéric Mortier, Eric Ragneau. New analytical method to evaluate the powerplant and chassis coupling in the improvement vehicle NVH. European Journal of Mechanics - A/Solids, 2005, 24, pp.929-943. 10.1016/j.euromechsol.2005.04.004 hal-00911592

\section{HAL Id: hal-00911592 \\ https://hal.science/hal-00911592}

Submitted on 29 Nov 2013

HAL is a multi-disciplinary open access archive for the deposit and dissemination of scientific research documents, whether they are published or not. The documents may come from teaching and research institutions in France or abroad, or from public or private research centers.
L'archive ouverte pluridisciplinaire HAL, est destinée au dépôt et à la diffusion de documents scientifiques de niveau recherche, publiés ou non, émanant des établissements d'enseignement et de recherche français ou étrangers, des laboratoires publics ou privés. 


\title{
New analytical method to evaluate the powerplant and chas- sis coupling in the improvement vehicle NVH
}

\author{
E. Courteille ${ }^{*+}$, L. Léotoing ${ }^{+}$, F. Mortier* and E. Ragneau ${ }^{+}$ \\ ${ }^{*}$ CF GOMMA Barre Thomas S.A \\ 194 Rte de Lorient 35043 RENNES \\ ${ }^{+}$Laboratoire de Génie Civil et Génie Mécanique (LGCGM) \\ INSA de RENNES \\ 20 Av des Buttes de Coësmes 35043 RENNES \\ e-mail: courteil@cfgomma.fr
}

\begin{abstract}
The design of an automotive powerplant mounting system plays an important role in improving vehicle noise, vibration and harshness (NVH). One of the main problems encountered in the automotive design remains the isolation of the low frequencies vibrations of the powerplant from the rest of the vehicle. Several powerplant mounting schemes have been developed to deal with this problem. Most of these strategies stem from arranging the rigid body modes of the powerplant mounted on resilient supports to have certain coupled or decoupled characteristics. It is currently known in literature that a decoupled powerplant mounting system improves NVH characteristics. The significant powerplant mass makes the right frequencies and modes arrangement a critical design decision. But it can not be stated that decoupling the grounded rigid body modes of the powerplant will systematically reduce chassis vibrations. In this paper, a new analytical method is proposed to examine the mechanisms of coupling between the powerplant and the vehicle body structure. The analytical procedure enables to define the domain of validity of the mounting schemes based on a 6 degrees-of-freedom powerplant model and to assess NVH improvement. As an example, the issue of the torque roll axis decoupling strategy is analyzed by this method in terms of improvement of the dynamic chassis responses at idle speed.
\end{abstract}

Keywords : powerplant mounting system, optimization, dynamic isolation, coupled systems 


\section{Introduction}

In vehicles, the engine mounts play an essential role for the NVH comfort. The main functions of these mounts (rubber or hydraulic) are to provide static supports for the powerplant and to isolate the vibrations of the powerplant from the rest of the vehicle. Thus, the modelling of these vehicle parts, becoming of a great complexity, constitutes an essential step for the NVH improvement. Besides, in addition to a good definition of the different vehicle parts, it is necessary to focus on the function of the subsystem in the whole vehicle by evaluating the main interactions with other components. Simulation of the powerplant mounting system at low frequency enables to define optimal architecture, and to give the characteristics of the engine mounts necessary in terms of rigidity and damping. But, to carry out an powerplant mounting system layout theoretically, system data have to be provided. As a good definition of the rest of the vehicle is not available in early stages of the vehicle design process, some assumptions have to be made. Specifically, the model includes rigid body representations of the powerplant and the chassis with appropriate values for the location of the centers of gravity, masses, and moments of inertia. Such a simulation model enables to assess the rigid body modes of the powerplant in the vehicle as well as to analyse the motion of the powerplant and the chassis under various engine operating conditions (idle, full load speed sweep) and road/wheel inputs.

Equations of motion for the powerplant engine mounting system include rigid chassis thought the chassis flexibility may have a significant effect on the powerplant vibration and the mounting forces transmitted from the powerplant to the structure, especially when flexible vibration modes of the chassis are excited. The dominant vibration modes of body structure at idle speed should be the first longitudinal bending mode and the first torsional mode, generally above 30-35 Hz. Future work should include the experimental verification of the simulation model assumptions through measurements of the vibration modes of the chassis.

The strategies currently used in industry analyze, under the modal approach, the harmonic response of the powerplant on resilient supports attached to ground [Brach, 1997, Khajepour and Geisberger, 2002]. The modal analysis of this six degrees of freedom (d.o.f.) model is interesting insofar as the response to an excitation is calculated and interpreted according to the position in frequency and to the form of the modes. The usual strategy moves the rigid body natural frequencies of the powerplant away from the frequencies of the input sources to avoid resonances [Gray et al., 1990, Kano and Hayashi, 1994]. By manipulating the rigid body modes of the grounded powerplant, the torque roll axis decoupling and the elastic axis decoupling methods attempt to shape the response with the aim of minimiz- 
ing the vibrations. The background theory of these techniques is widely described in literature [Patton and Geck, 1984, Singh and Jeong, 2000, Brach, 1997]. All these studies consider the powerplant by its grounded behavior, neglecting the effects of the chassis, exhaust subsystem, drive-shaft, wheel suspension ...

Lately, researches have focused on the significance of the rigid body modes alignment for grounded powerplant to its in-vehicle behavior [Sirafi and Qatu, 2003, Hadi and Sachdeva, 2003]. These studies deal with the accuracy of NVH vehicle models and raise the problem of interactions between the different subsystems. Various powertrain models have been studied and their accuracy was discussed through a full vehicle model. By the evaluation of actual cases, the existence of these interactions have been clearly demonstrated. Nevertheless, no general formalism have been introduced to evaluate the limits of the modelling assumptions made during the development of the classical 6 d.o.f. powerplant mounting schemes.

The aim of the proposed method is to highlight and identify, through an analytical procedure, the relationships between the powerplant mounting schemes and the vehicle response characteristics at idle speed. In the second section, the general equations of motion are reformulated using an original matrix, the coupling matrix introduced for coupled plates [Bessac F. and Guyader, 1996]. The characteristics of the coupling matrix, analyzed in the third section, enable to define the domain of validity of the mounting schemes based on a 6 d.o.f. powerplant model and to assess NVH improvement. In the last section, the issue of the torque roll axis decoupling strategy is analyzed by this method in terms of improvement of the dynamic chassis responses.

\section{FORMULATION OF THE COUPLING PROBLEM}

\subsection{Modelling of the vehicle system}

In derivation of the equations of motion to simulate dynamic behaviors of powerplant mounting systems with supporting structures, a good modelling of a total vehicle system can consist of four subsystems : powerplant which includes engine and transmission, engine mounts, chassis and suspensions. Since small displacements can be assumed, powerplant is modelled as rigid body of time-invariant inertial matrix of dimension 6 . The powerplant is supported by an arbitrary number of mounts to the vehicle chassis, also modelled as a rigid body elastically suspended (Figure 1).

The mounts classically used in powerplant mounting application are rubber bonded to metal construction. It is possible to get better isolation effects than conventional rubber mount systems with 
a hydraulic engine mount. Hydraulic engine mount control the damping characteristics by using the fluid viscosity. Elastomeric materials behave visco-elastically, thus engine mounts are represented by three mutually perpendicular sets of linear spring and corresponding viscous damper in parallel. No rotational stiffness of the mounts will be considered. The stiffness matrix $\mathbf{K}_{m i}$ and damping matrix $\mathbf{C}_{m i}$ of a mount $i$ can be written in its local coordinate system as :

$$
\mathbf{K}_{m i}=\left[\begin{array}{ccc}
k_{u} & 0 & 0 \\
0 & k_{v} & 0 \\
0 & 0 & k_{w}
\end{array}\right]_{m i} \text {, and } \mathbf{C}_{m i}=\left[\begin{array}{ccc}
c_{u} & 0 & 0 \\
0 & c_{v} & 0 \\
0 & 0 & c_{w}
\end{array}\right]_{m i}
$$

The subscript $m i$ stands for the mount frame coordinates $\mathcal{R}_{m i}$ (Figure 1 ) of the the $i^{\text {th }}$ mount. The stiffness and damping matrices must be transformed from the local mount coordinate system $\mathcal{R}_{m i}$ to the global coordinate system $\mathcal{R}$ by the following linear transformation :

$$
\mathbf{K}_{i}=\boldsymbol{\Pi}_{m i}^{-1} \mathbf{K}_{m i} \boldsymbol{\Pi}_{m i}=\left[\begin{array}{ccc}
k_{x x} & k_{x y} & k_{x z} \\
k_{x y} & k_{y y} & k_{y z} \\
k_{x z} & k_{y z} & k_{z z}
\end{array}\right] \text {, and } \mathbf{C}_{i}=\mathbf{\Pi}_{m i}^{-1} \mathbf{C}_{m i} \boldsymbol{\Pi}_{m i}=\left[\begin{array}{ccc}
c_{x x} & c_{x y} & c_{x z} \\
c_{x y} & c_{y y} & c_{y z} \\
c_{x z} & c_{y z} & c_{z z}
\end{array}\right]
$$

where $\boldsymbol{\Pi}_{m i}$ is the transformation matrix from the local coordinate system $\mathcal{R}_{m i}$ to the global one $\mathcal{R}$. The elements of $\boldsymbol{\Pi}_{m i}$ consist of directional cosines of the local frame with respect to $\mathcal{R}$ defined from Euler angles.

\subsection{Equations of motion}

In order to express the equations of motion in terms of displacements and rotations of the powerplant and chassis centers of gravity, another transformation is necessary. This transformation relates the displacements of each mount to the displacements and rotations of the powerplant and chassis centers of gravity. The superscripts $(e)$ and $(c)$ respectively stand for powerplant and chassis. The superscript (b) may refer to either the powerplant or the chassis. A generalized vector $\mathbf{q}$ (Eq. 3) is defined by combining translational $\mathbf{u}$ and rotational $\boldsymbol{\theta}$ displacements of the centers of gravity of the powerplant (Figure 2) and of the chassis.

$$
\mathbf{q}={ }^{t}\left\{\mathbf{q}^{(e)} \quad \mathbf{q}^{(c)}\right\}={ }^{t}\left\{\begin{array}{llll}
\mathbf{u}^{(e)} & \boldsymbol{\theta}^{(e)} & \mathbf{u}^{(c)} & \boldsymbol{\theta}^{(c)}
\end{array}\right\}
$$

The position vector of the $i^{t h}$ mount's center of elasticity with respect to the center of gravity of the powerplant and the chassis are given in terms of global coordinate system as : 


$$
\mathbf{r}_{i}^{(b)}={ }^{t}\left\{x_{i}^{(b)} \quad y_{i}^{(b)} \quad z_{i}^{(b)}\right\}
$$

and each has a corresponding skew asymmetric matrix defined as :

$$
\mathbf{A}_{i}^{(b)}=\left[\begin{array}{ccc}
0 & z_{i}^{(b)} & -y_{i}^{(b)} \\
-z_{i}^{(b)} & 0 & x_{i}^{(b)} \\
y_{i}^{(b)} & -x_{i}^{(b)} & 0
\end{array}\right]
$$

with a generalized form :

$$
\mathbf{P}_{i}^{(b)}=\left[\begin{array}{ll}
\mathbf{I} & \mathbf{A}_{i}^{(b)}
\end{array}\right]
$$

Let be $\mathbf{u}_{i}^{(b)}$ the translational displacement vector at the mounting point $i$ for the rigid body $(b)$ side. The relative translational displacement vector $\boldsymbol{\delta}_{i}$ for the $i^{\text {th }}$ mount for small motions is related to the rigid body center of gravity motions and the translational displacements at the mounting point according to the equation (7).

$$
\boldsymbol{\delta}_{i}=\left(\mathbf{u}_{i}^{(e)}-\mathbf{u}_{i}^{(c)}\right)=\left(\mathbf{u}^{(e)}-\mathbf{u}^{(c)}\right)+\left(\mathbf{A}_{i}^{(e)} \boldsymbol{\theta}^{(e)}-\mathbf{A}_{i}^{(c)} \boldsymbol{\theta}^{(c)}\right)
$$

The translational reaction force $\mathbf{f}_{i}^{(e)}$ and $\mathbf{f}_{i}^{(c)}$ and moment reaction $\boldsymbol{\tau}_{i}^{(e)}$ and $\boldsymbol{\tau}_{i}^{(c)}$ resulting from the application of the elastic forces of mounting $i$ respectively on the powerplant and the chassis centers of gravity can be expressed in the $\mathcal{R}$ frame as :

$$
\begin{aligned}
\mathbf{f}_{i}^{(e)} & =-\mathbf{K}_{i} \boldsymbol{\delta}_{i}=-\mathbf{K}_{i}\left({ }^{t} \mathbf{P}_{i}^{(e)} \mathbf{q}^{(e)}-{ }^{t} \mathbf{P}_{i}^{(c)} \mathbf{q}^{(c)}\right) \\
\mathbf{f}_{i}^{(c)} & =-\mathbf{f}_{i}^{(e)} \\
\boldsymbol{\tau}_{i}^{(e)} & ={ }^{t} \mathbf{A}_{i}^{(e)} \mathbf{f}_{i}^{(e)}=\mathbf{A}_{i}^{(e)} \mathbf{K}_{i}\left({ }^{t} \mathbf{P}_{i}^{(e)} \mathbf{q}^{(e)}-{ }^{t} \mathbf{P}_{i}^{(c)} \mathbf{q}^{(c)}\right) \\
\boldsymbol{\tau}_{i}^{(c)} & ={ }^{t} \mathbf{A}_{i}^{(c)} \mathbf{f}_{i}^{(c)}=-\mathbf{A}_{i}^{(c)} \mathbf{K}_{i}\left({ }^{t} \mathbf{P}_{i}^{(e)} \mathbf{q}^{(e)}-{ }^{t} \mathbf{P}_{i}^{(c)} \mathbf{q}^{(c)}\right)
\end{aligned}
$$

At idle speed, the connection to the ground is simply represented by four systems of linear spring and viscous damper in parallel at each wheel, characterized by their stiffness and damping coefficients following the three directions of the vehicle frame coordinates $\mathcal{R}$. The translational reaction force $\mathbf{f}_{k}^{(c)}$ and moment reaction $\boldsymbol{\tau}_{k}^{(c)}$ from the $k^{\text {th }}$ suspension applied to the chassis can be expressed in the $\mathcal{R}$ frame with the displacement of the chassis $\mathbf{u}_{k}^{(c)}$ at the supporting point as : 


$$
\begin{aligned}
\mathbf{f}_{k}^{(c)} & =-\mathbf{K}_{k} \mathbf{u}_{k}^{(c)}=-\mathbf{K}_{k}{ }^{t} \mathbf{P}_{k}^{(c)} \mathbf{q}^{(c)} \\
\boldsymbol{\tau}_{k}^{(c)} & ={ }^{t} \mathbf{A}_{k}^{(c)} \mathbf{f}_{k}^{(c)}=\mathbf{A}_{k}^{(c)} \mathbf{K}_{k}{ }^{t} \mathbf{P}_{k}^{(c)} \mathbf{q}^{(c)}
\end{aligned}
$$

For road/wheel inputs, similarly a simple model can be used for the wheel-suspension system, with a single degree of freedom. It can be represented by a mass and a spring accounting for the wheel mass and the tire's stiffness in parallel with a spring and a damper accounting for the suspension system. Future work should include the study of the dynamic interaction between the vehicle suspensions and the powerplant mounting system.

By assuming all of the elastic loadings from all mounts and suspensions, the total elastic loadings on the powerplant and chassis centers of gravity can be expressed through a generalized square stiffness matrix $\mathbf{K}$ of dimension 12 (10), resulting from the assembly of the stiffness elementary matrices (mounts and suspensions).

$$
\mathbf{K}=\left[\begin{array}{cc}
\mathbf{K}^{(e)} & -\mathbf{K}^{(e \rightarrow c)} \\
-\mathbf{K}^{(c \rightarrow e)} & \mathbf{K}^{(c)}
\end{array}\right]=\left[\begin{array}{cc}
\sum_{i} \mathbf{P}_{i}^{(e)} \mathbf{K}_{i}{ }^{t} \mathbf{P}_{i}^{(e)} & -\sum_{i} \mathbf{P}_{i}^{(e)} \mathbf{K}_{i}{ }^{t} \mathbf{P}_{i}^{(c)} \\
-\sum_{i} \mathbf{P}_{i}^{(c)} \mathbf{K}_{i}{ }^{t} \mathbf{P}_{i}^{(e)} & \sum_{i} \mathbf{P}_{i}^{(c)} \mathbf{K}_{i}{ }^{t} \mathbf{P}_{i}^{(c)}+\sum_{k} \mathbf{P}_{k}^{(c)} \mathbf{K}_{k}{ }^{t} \mathbf{P}_{k}^{(c)}
\end{array}\right]
$$

The matrix $\mathbf{K}^{(e \rightarrow c)}$ is the powerplant's matrix of influence on the chassis and reciprocally, $\mathbf{K}^{(c \rightarrow e)}$ is the chassis's matrix of influence on the powerplant. Using a similar assembly procedure to the elastic loadings, the total damping loadings on the powerplant and chassis centers of gravity can be expressed by a generalized square stiffness damping matrix $\mathbf{C}$ of dimension 12 (11).

$$
\mathbf{C}=\left[\begin{array}{cc}
\mathbf{C}^{(e)} & -\mathbf{C}^{(e \rightarrow c)} \\
-\mathbf{C}^{(c \rightarrow e)} & \mathbf{C}^{(c)}
\end{array}\right]=\left[\begin{array}{cc}
\sum_{i} \mathbf{P}_{i}^{(e)} \mathbf{C}_{i}{ }^{t} \mathbf{P}_{i}^{(e)} & -\sum_{i} \mathbf{P}_{i}^{(e)} \mathbf{C}_{i}{ }^{t} \mathbf{P}_{i}^{(c)} \\
-\sum_{i} \mathbf{P}_{i}^{(c)} \mathbf{C}_{i}{ }^{t} \mathbf{P}_{i}^{(e)} & \sum_{i} \mathbf{P}_{i}^{(c)} \mathbf{C}_{i}{ }^{t} \mathbf{P}_{i}^{(c)}+\sum_{k} \mathbf{P}_{k}^{(c)} \mathbf{C}_{k}{ }^{t} \mathbf{P}_{k}^{(c)}
\end{array}\right]
$$

With all of the component reactive forces derived in terms of the generalized coordinates, and under the assumption of small oscillations the equations of motion of the powerplant and the car body structure can be written as the matrix form in the frequency domain:

$$
\left(-\omega^{2} \mathbf{M}+j \omega \mathbf{C}+\mathbf{K}\right) \mathbf{q}(\omega)=\mathbf{F}(\omega)
$$

The vector $\mathbf{F}={ }^{t}\left\{\begin{array}{ll}\mathbf{F}^{(e)} & \mathbf{F}^{(c)}\end{array}\right\}$ is the generalized external load vector. The external excitation is harmonic with known frequencies, amplitudes and phases. Engine excitation forces are applied to the powerplant at the center of the crankshaft location. The response to road inputs can be studied 
by applying forces or displacements at the suspension ground contact locations in the vehicle model. The matrix $\mathbf{M}$ is the generalized mass matrix of the system (13).

$$
\begin{aligned}
\mathbf{M} & =\left[\begin{array}{cc}
\mathbf{M}^{(e)} & \mathbf{0} \\
\mathbf{0} & \mathbf{M}^{(c)}
\end{array}\right]=\left[\begin{array}{cccc}
\mathbf{M}_{f}^{(e)} & \mathbf{0} & \mathbf{0} & \mathbf{0} \\
\mathbf{0} & \mathbf{M}_{\tau}^{(e)} & \mathbf{0} & \mathbf{0} \\
\mathbf{0} & \mathbf{0} & \mathbf{M}_{f}^{(c)} & \mathbf{0} \\
\mathbf{0} & \mathbf{0} & \mathbf{0} & \mathbf{M}_{\tau}^{(c)}
\end{array}\right] \\
\text { with } \mathbf{M}_{f}^{(b)} & =\left[\begin{array}{ccc}
m^{(b)} & 0 & 0 \\
0 & m^{(b)} & 0 \\
0 & 0 & m^{(b)}
\end{array}\right] \text { and } \mathbf{M}_{\tau}^{(b)}=\left[\begin{array}{ccc}
I_{x x}^{(b)} & -I_{x y}^{(b)} & -I_{x z}^{(b)} \\
-I_{x y}^{(b)} & I_{y y}^{(b)} & -I_{y z}^{(b)} \\
-I_{x z}^{(b)} & -I_{y z}^{(b)} & I_{z z}^{(b)}
\end{array}\right]
\end{aligned}
$$

$m^{(b)}$ is the mass of the rigid body $(b)$ and $\mathbf{M}_{\tau}^{(b)}$ its inertia matrix. $\mathbf{C}$ is the generalized viscous damping matrix assuming a proportionally damped system. If a structural damping matrix $\mathbf{H}$ is considered, viscous damping term $j \omega \mathbf{C}$ may be replaced by the structural damping term $j \mathbf{H}$. For the following developments, a complex stiffness is used to model the dynamic behavior of the isolators. The bar indicates that the stiffness term is complex $(\overline{\mathbf{K}}=\mathbf{K}+j \omega \mathbf{C}$ or $\overline{\mathbf{K}}=\mathbf{K}+j \mathbf{H})$.

\subsection{Introduction of the coupling matrix}

The response of the powerplant and chassis centers of gravity can be calculated through the solving of equation (12). Then the complex matrix inversion of equation (14) is classically used.

$$
\left\{\begin{array}{c}
\mathbf{q}^{(e)} \\
\mathbf{q}^{(c)}
\end{array}\right\}=\left[\begin{array}{cc}
\left(\overline{\mathbf{K}}^{(e)}-\omega^{2} \mathbf{M}^{(e)}\right) & -\overline{\mathbf{K}}^{(e \rightarrow c)} \\
-\overline{\mathbf{K}}^{(c \rightarrow e)} & \left(\overline{\mathbf{K}}^{(c)}-\omega^{2} \mathbf{M}^{(c)}\right)
\end{array}\right]^{-1}\left\{\begin{array}{c}
\mathbf{F}^{(e)} \\
\mathbf{F}^{(c)}
\end{array}\right\}
$$

The inversion of the matrix of impedance is numerically commonplace. Nevertheless, this method of resolution prevent from understanding the coupling phenomena between the powerplant and the chassis. From the traditional equation of motion (14), one can isolate a matrix presenting only terms related to the coupling from the two bodies (15).

$$
\left[\begin{array}{cc}
\mathbf{I} & -\left(\overline{\mathbf{K}}^{(e)}-\omega^{2} \mathbf{M}^{(e)}\right)^{-1} \overline{\mathbf{K}}^{(e \rightarrow c)} \\
-\left(\overline{\mathbf{K}}^{(c)}-\omega^{2} \mathbf{M}^{(c)}\right)^{-1} \overline{\mathbf{K}}^{(c \rightarrow e)} & \mathbf{I}
\end{array}\right]\left\{\begin{array}{c}
\mathbf{q}^{(e)} \\
\mathbf{q}^{(c)}
\end{array}\right\}=\left\{\begin{array}{c}
\left(\overline{\mathbf{K}}^{(e)}-\omega^{2} \mathbf{M}^{(e)}\right)^{-1} \mathbf{F}^{(e)} \\
\left(\overline{\mathbf{K}}^{(c)}-\omega^{2} \mathbf{M}^{(c)}\right)^{-1} \mathbf{F}^{(c)}
\end{array}\right\}
$$

For the sake of physical meaning of the coupling mechanism, the term $\left(\overline{\mathbf{K}}^{(e)}-\omega^{2} \mathbf{M}^{(e)}\right)^{-1} \mathbf{F}^{(e)}$ in equation (15) represents the displacement of the powerplant subjected to its own excitation when the chassis is blocked (suspensions with infinite stiffnesses). This configuration represents the 
grounded behavior of the powerplant (Figure 3-(a)). The term $\left(\overline{\mathbf{K}}^{(c)}-\omega^{2} \mathbf{M}^{(c)}\right)^{-1} \mathbf{F}^{(c)}$ represents the displacement of the chassis subjected to his own excitation when the powerplant is blocked (null displacements) (Figure 3-(b)). This configuration however do not represent a realistic behavior. One expresses the two configurations by the generalized vector displacement of the blocked uncoupled $\operatorname{bodies}^{t}\left\{\begin{array}{ll}\mathbf{q}_{0}^{(e)} & \mathbf{q}_{0}^{(c)}\end{array}\right\}(16)$.

$$
\left\{\begin{array}{c}
\mathbf{q}_{0}^{(e)} \\
\mathbf{q}_{0}^{(c)}
\end{array}\right\}=\left\{\begin{array}{c}
\left(\overline{\mathbf{K}}^{(e)}-\omega^{2} \mathbf{M}^{(e)}\right)^{-1} \mathbf{F}^{(e)} \\
\left(\overline{\mathbf{K}}^{(c)}-\omega^{2} \mathbf{M}^{(c)}\right)^{-1} \mathbf{F}^{(c)}
\end{array}\right\}
$$

While revealing the vector displacement of the coupled systems, the equation (15) takes a form such that a coupling matrix D appears [Bessac F. and Guyader, 1996] (17).

$$
\begin{gathered}
\left\{\begin{array}{c}
\mathbf{q}^{(e)} \\
\mathbf{q}^{(c)}
\end{array}\right\}=\left\{\begin{array}{c}
\mathbf{q}_{0}^{(e)} \\
\mathbf{q}_{0}^{(c)}
\end{array}\right\}+\mathbf{D}\left\{\begin{array}{c}
\mathbf{q}^{(e)} \\
\mathbf{q}^{(c)}
\end{array}\right\} \\
\text { with } \mathbf{D}=\left[\begin{array}{c}
\mathbf{0} \\
\left.\left(\overline{\mathbf{K}}^{(c)}-\omega^{2} \mathbf{M}^{(c)}\right)^{-1} \overline{\mathbf{K}}^{(c \rightarrow e)}\left(\overline{\mathbf{K}}^{(e)}-\omega^{2} \mathbf{M}^{(e)}\right)^{-1} \overline{\mathbf{K}}^{(e \rightarrow c)}\right]
\end{array}\right.
\end{gathered}
$$

Each one of the coupling matrix terms represents the action of the powerplant mass displacement (respectively chassis) on the chassis mass displacement (respectively powerplant). The matrix of coupling describes the exchanges between the masses independently of the external excitation. The coupling matrix, studied in more details in the following part, is then a practical solution to predict the global behavior of a system starting from the behavior of the isolated subsystems.

\section{COUPLING MATRIX}

This section is dedicated to the analysis of the coupling matrix by extracting its eigenvalues. The value of these intrinsic characteristics permits to identify different zone in which the degree of coupling between the powerplant and the chassis can be evaluated through an original parameter : the coupling order.

\subsection{Eigenvalues and eigenvectors of coupling}

The eigenvalues of coupling and the eigenvectors of coupling can be extracted from the coupling matrix. Equation (18) formulate the eigenvalue problem associated with the eigenvalues of coupling $\lambda_{r}(\omega)(r=1, . ., 12)$ and the eigenvectors of coupling $\varphi_{r}(\omega)$. 


$$
\operatorname{det}\left(\mathbf{D}-\lambda_{r}(\omega) \mathbf{I}\right)=0
$$

Figure 4 presents the evolution of the spectral radius of the coupling matrix $\mathbf{D}$ versus frequency for three representative models of front wheel drive cars (a 3, 4 and 6 cylinder engine, tables 1 and 2). The spectral radius of $\mathbf{D}$ is defined by $\rho(\mathbf{D})=\max _{r}\left|\lambda_{r}\right|$. One can see in this figure that resonant peaks appear for the spectral radius and at the resonance frequencies of the uncoupled blocked bodies (Table 3). This property of the spectral radius of $\mathbf{D}$ is clearly demonstrated by rewriting the determinant of the coupling matrix (19).

$$
\operatorname{det} \mathbf{D}=\frac{\operatorname{det} \overline{\mathbf{K}}^{(e \rightarrow c)} \operatorname{det} \overline{\mathbf{K}}^{(c \rightarrow e)}}{\operatorname{det}\left(\overline{\mathbf{K}}^{(e)}-\omega^{2} \mathbf{M}^{(e)}\right) \operatorname{det}\left(\overline{\mathbf{K}}^{(c)}-\omega^{2} \mathbf{M}^{(c)}\right)}=\prod_{r} \lambda_{r}
$$

At the resonance frequencies of the uncoupled blocked bodies, $\left(\operatorname{det}\left(\overline{\mathbf{K}}^{(e)}-\omega^{2} \mathbf{M}^{(e)}\right)\right)$ or $\left(\operatorname{det}\left(\overline{\mathbf{K}}^{(c)}-\omega^{2} \mathbf{M}^{(c)}\right)\right)$ is null, then the coupling eigenvalues product and respectively the spectral radius become infinite. The increase in the damping of the connections smoothes these resonant peaks. Moreover, the frequencies of the peaks are located in the frequency band defined by the eigenvalues of the coupled bodies [Julien, 1960] (Table 4). To support the powerplant weight and to avoid interference between the powerplant and the chassis during limit running conditions such as bumps and sudden brakes, a minimum level of stiffness is necessary for the engine mounts. The frequency range of the rigid body modes of suspension are then located between 3 and $20 \mathrm{~Hz}$ according to the mass of the powerplant.

\subsection{Hypothesis on the equations of motion}

The coupling matrix describes the contribution of one system on the other. One rewrites the equation (17) connecting the displacement of the systems coupled with the displacement of the blocked uncoupled bodies (20).

$$
\left\{\begin{array}{c}
\mathbf{q}^{(e)} \\
\mathbf{q}^{(c)}
\end{array}\right\}=(\mathbf{I}-\mathbf{D})^{-1}\left\{\begin{array}{c}
\mathbf{q}_{0}^{(e)} \\
\mathbf{q}_{0}^{(c)}
\end{array}\right\}
$$

The components resulting from the excitation $\left(\mathbf{q}_{0}^{(b)}\right)$ are isolated from the terms of the coupling matrix, intrinsic with the phenomena of coupling. If $\|\mathbf{D}\|<1$, where $\|$.$\| is the Frobenius norm or$ one of the $p$-norms, then $(\mathbf{I}-\mathbf{D})$ is nonsingular and : 


$$
(\mathbf{I}-\mathbf{D})^{-1}=\sum_{n=0}^{\infty} \mathbf{D}^{n} .
$$

Thus, the equation (20) can be written as follow while $\|\mathbf{D}\|<1$ :

$$
\left\{\begin{array}{c}
\mathbf{q}^{(e)} \\
\mathbf{q}^{(c)}
\end{array}\right\}=\sum_{n=0}^{\infty} \mathbf{D}^{n}\left\{\begin{array}{c}
\mathbf{q}_{0}^{(e)} \\
\mathbf{q}_{0}^{(c)}
\end{array}\right\}
$$

The spectral radius of the 12-by-12 matrix of coupling $\mathbf{D}$, defined in the previous section, gives the lower bound of the all norms matrix of $\mathbf{D}(23)$.

$$
\rho(\mathbf{D}) \leq\|\mathbf{D}\| \leq 1
$$

As shown before, the resonant peaks of the spectral radius appear at the resonance frequencies of the uncoupled blocked bodies (Figure 4), i.e. between 3 and $16 \mathrm{~Hz}$ (Table 4). The figure 5 shows that beyond $16 \mathrm{~Hz}$, the value of the spectral radius decrease gradually for tending toward zero. From (23), the development (22) can only be valid apart from resonant peaks of the coupling eigenvalues, i.e. apart from the domain of appearance of the eigenvalues of the blocked uncoupled bodies. As presented in figure 5, the assumption is valid from approximately $22 \mathrm{~Hz}$ for the 4 and 6 cylinder engine models. For the 3 cylinder engine model, the assumption is valid from approximately $30 \mathrm{~Hz}$ because the resonance frequencies of the uncoupled blocked bodies are slightly higher.

\subsection{Coupling order}

In an adequate interval of frequencies, i.e. when the value of the norm of the coupling matrix is negligible in front of the unit, the development of equation (22) can be restricted to a weaker order $(24)$.

$$
\left\{\begin{array}{c}
\mathbf{q}^{(e)} \\
\mathbf{q}^{(c)}
\end{array}\right\} \approx \sum_{n=0}^{N} \mathbf{D}^{n}\left\{\begin{array}{c}
\mathbf{q}_{0}^{(e)} \\
\mathbf{q}_{0}^{(c)}
\end{array}\right\}
$$

The parameter $N$ is called «coupling order $\gg$, it expresses the coupling level between the two rigid bodies for the domain of frequency studied. The evaluation of $N$ is essential for the global analysis in order to obtain simplistic relations between coupled system displacements and blocked uncoupled bodies ones. The last part of this paper notably illustrates the interests of low coupling orders.

The traditional mounting system design strategies require the rigid body modes of the grounded engine. Theses strategies involve only powerplant rigid body mode arrangements for "shaping" 
the powerplant vibratory behavior, i.e. the vector $\mathbf{q}_{0}^{(e)}$. According to the domain of frequency studied, the phenomena of interactions between rigid bodies can drastically modify the vibratory responses of the vehicle. The internal vibroacoustic comfort in automobiles is directly controlled by the chassis acceleration. In a preliminary design phase, a 6 d.o.f. model is indispensable to conduct an powerplant rigid body mode analysis. But a higher level NVH model that contains powerplant and chassis on suspension should be used to understand the interactions between the rigid bodies thanks to the general expression (17) or to the expression (24) valid in the isolation band, where $\|\mathbf{D}\|<1$. These expressions enable to examine the domain of validity of the modelling assumptions and profit towards classical powerplant mounting strategies on the NVH improvement. By way of example, using the proposed method, the following part analyses the issue of the torque roll axis decoupling, strategy often used for the idle isolation.

\section{DYNAMIC DECOUPLING METHOD}

As a rigid body, the powerplant presents six modes of vibrations on its mounts. In the automotive industry they are commonly referred to bounce, lateral, and longitudinal for the translation modes and roll, pitch, and yaw for the rotational modes. Roll mode is the rotation about the $Y$ axis of the $\mathcal{R}$ frame, pitch about the $X$ axis and yaw the rotation about the $Z$ axis.

\subsection{Torque roll axis decoupling concepts}

It is hypothesized that the disturbances transferred to the car body structure can be reduced by conditioning the powerplant mounting system such that the powerplant oscillates about the torque axis [Shane Sui and Hoppe, 2003]. The torque axis, the so-called torque roll axis (TRA), is defined as the resulting fixed axis of rotation of an slightly constrained three dimensional rigid body (i.e. either free or supported elastically on very soft springs) when a torque is applied along any axis. In first approximation for the automotive case, the axis about which the pulsating torque generated by the transversal multi-cylinder engines at idle is applied is the crankshaft axis. Geck and Patton [Patton and Geck, 1984] give a mathematical proof for the conditions which ensure that the engine pulsating torque excites only one powerplant mode. Singh and Jeong [Singh and Jeong, 2000] demonstrate from the axioms of Geck and Patton that when the constant direction of the response becomes a rigid body mode of the grounded powerplant, then the response is a rotation around the $T R A$, so with a constant direction. The harmonic torque $\mathbf{T}(\omega)$ is applied along the crankshaft axis which in a front wheel drive car coincides with $Y$ axis of the $\mathcal{R}$ frame (25). 


$$
\mathbf{T}(\omega)=\mathbf{T}_{A} e^{j \omega t}={ }^{t}\left\{\begin{array}{llllll}
0 & 0 & 0 & 0 & T_{y} & 0
\end{array}\right\} e^{j \omega t}
$$

The $T R A$ direction $\mathbf{q}_{T R A}$ is only defined by the mass matrix of the powerplant (2.2) and the torque direction (25) [Singh and Jeong, 2000] :

$$
\mathbf{q}_{T R A}=a\left(\mathbf{M}^{(e)}\right)^{-1} \mathbf{T}_{A}
$$

where $a$ is a constant for the vector normalization. For common front wheel drive engine, the $T R A$ is seldom parallel to the inertia axis. By convenience of simplicity, the assumption that the principle inertia axis of the powerplant are parallel to the coordinate axis of the $\mathcal{R}$ frame is made. Thus $\mathbf{q}_{T R A}={ }^{t}\left\{\begin{array}{llllll}0 & 0 & 0 & 0 & 1 & 0\end{array}\right\}$. Thanks to this approximation, the torque axis mounting strategy consists in a simple rotation decoupling in the elastic axes with a control of the eigenvalue. Elastic axes for an elastically supported rigid body system are those axes for which application of a force or torque, along or about an axe produces only a corresponding translation or rotation on or about the same line. If all the elastic axes coincide with the principal inertia axes, the rigid body response consists of decoupled translational and rotational modes. To achieve the torque axis mounting strategy, the TRA direction should be one of the rigid body modes of the powerplant. The equation (27) must be satisfied.

$$
\overline{\mathbf{K}}^{(e)} \mathbf{q}_{T R A}=\omega_{T R A} \mathbf{M}^{(e)} \mathbf{q}_{T R A},
$$

where $\omega_{T R A}$ is the eigenvalue of the decoupled mode. The system of equations (27), once developed (28), needs to be satisfied for a complete decoupling of the TRA mode.

$$
\left\{\begin{aligned}
\sum_{i}\left(\bar{k}_{x x i} z_{i}^{(e)}-\bar{k}_{x z i} x_{i}^{(e)}\right) & =0 \\
\sum_{i}\left(\bar{k}_{x y i} z_{i}^{(e)}-\bar{k}_{y z i} x_{i}^{(e)}\right) & =0 \\
\sum_{i}\left(\bar{k}_{x z i} z_{i}^{(e)}-\bar{k}_{z z i} x_{i}^{(e)}\right) & =0 \\
\sum_{i}\left(-\bar{k}_{x y i}\left(z_{i}^{(e)}\right)^{2}+\bar{k}_{y z i} x_{i}^{(e)} z_{i}^{(e)}+\bar{k}_{x z i} z_{i}^{(e)} y_{i}^{(e)}-\bar{k}_{z z i} x_{i}^{(e)} y_{i}^{(e)}\right) & =0 \\
\sum_{i}\left(\bar{k}_{x x i}\left(z_{i}^{(e)}\right)^{2}-2 \bar{k}_{x z i} x_{i}^{(e)} z_{i}^{(e)}+\bar{k}_{z z i}\left(x_{i}^{(e)}\right)^{2}\right) & =-\omega_{T R A} I_{y y}^{(e)} \\
\sum_{i}\left(-\bar{k}_{x x i} z_{i}^{(e)} y_{i}^{(e)}+\bar{k}_{x z i} x_{i}^{(e)} y_{i}^{(e)}+\bar{k}_{x y i} z_{i}^{(e)} x_{i}^{(e)}-\bar{k}_{y z i}\left(x_{i}^{(e)}\right)^{2}\right) & =0
\end{aligned}\right.
$$

The torque roll axis decoupling strategy controls the displacement of the uncoupled blocked powerplant. The grounded powerplant has a frequency response only in the $T R A$ direction with the 
crankshaft torque variation excitation in all frequency range $\left(\mathbf{q}_{0}^{(e)}={ }^{t}\left\{\begin{array}{llllll}0 & 0 & 0 & 0 & \theta_{y}^{(e)}(\omega) & 0\end{array}\right\}\right)$. Only one resonance mode frequency, which is the $T R A$ mode frequency, is excited (Figure 6). In the decoupled configuration, the grounded powerplant response can easily be expressed by equation (29). There is only one frequency to move away from the excitation frequency to reduce powerplant vibrations.

$$
\theta_{0 y}^{(e)}=\frac{T_{y}}{I_{y y}^{(e)}\left(\omega_{T R A}^{2}-\omega^{2}\right)}
$$

The design objective is to reduce vehicle vibration in certain frequency range with respect to idle engine excitations. At idle, only the powerplant is directly excited, so the uncoupled blocked chassis has no displacements $\left(\mathbf{F}^{(c)}=0\right.$ then $\left.\mathbf{q}_{0}^{(c)}=\mathbf{0}\right)$. However the vibratory behavior of the vehicle cannot be limited to a study restricted with this six d.o.f model.

\subsection{Evaluation of the coupling order}

For a 3-cylinder engine, the inputs are particularly low in frequency at idle; the combustion forces are 1.5 order $(17.5 \mathrm{~Hz}$ at $700 \mathrm{rpm})$ and the unbalance moments are first order $(11.6 \mathrm{~Hz}$ at 700 $\mathrm{rpm})$. The fundamental frequency of the excitation is in the frequency range of the suspension modes, where the coupling eigenvalues present resonant peaks. Therefore, the interaction between the rigid bodies is high (Figures 5 and 4 ) and the couplings with the chassis will strongly modify the vibratory response of the powerplant. The interest of the torque roll axis decoupling strategy for a 3-cylinder engine, already applied by Saitoh [Saitoh and Igarashi, 1990], is not at all obvious because the powerplant behavior in the vehicle can be very different from that on the ground (Figure 7, up to $15 \mathrm{~Hz}$ ). Note that for a 3 cylinder engine, the unbalance inputs about the pitch and yaw axes can be as high as the oscillating torque due to the combustion forces. A numerical analysis must be done by the equation (17) to obtain the hypothetic benefits on the chassis response by a purification of the powerplant vibratory response.

This is in contrast with 4 and 6 cylinder engines for which an evaluation of the coupling order can lead to an analytical study of the coupling phenomena. The fundamental frequency of the excitation is in the zone of filtering, beyond the frequencies of the rigid body modes. Indeed, at the second order, i.e. $25 \mathrm{~Hz}$ for a 4 cylinder engine at $750 \mathrm{rpm}$, and at the third order, i.e. $40 \mathrm{~Hz}$ for a 6 cylinder engine at $800 \mathrm{rpm}$, the value of the norm of the coupling matrix is small in front of the unit (Figure 5).

The figure 8 represents the decoupled frequency response of the powerplant center of gravity of the 
4 cylinder engine model for different values of the coupling order $N$. The powerplant response at the first order development, which corresponds to the blocked uncoupled powerplant response $\mathbf{q}_{0}^{(e)}$ because of the form extra-diagonal of $\mathbf{D}$, is quite different from the in-vehicle powerplant response $(N \longrightarrow \infty)$. Then the development can be restricted to the second order for the powerplant displacement (Figure 8 ) since the difference between the two plots $(N=2)$ and $(N \longrightarrow \infty)$ never exceeds $1 \%$. In the same way, the figure 9 represents the decoupled frequency response of the chassis center of gravity for the 4 cylinder engine model for different values of $N$. Then, for the frequency domain studied, a first coupling order leads to a good approximation of the chassis response. For the global system, a second coupling order is therefore sufficient :

$$
\left\{\begin{array}{c}
\mathbf{q}^{(e)} \\
\mathbf{q}^{(c)}
\end{array}\right\} \approx\left(\mathbf{I}+\mathbf{D}+\mathbf{D}^{2}\right)\left\{\begin{array}{c}
\mathbf{q}_{0}^{(e)} \\
\mathbf{q}_{0}^{(c)}
\end{array}\right\}
$$

According to the equation (30), with $\mathbf{q}_{0}^{(c)}=\mathbf{0}$, the chassis displacements are given by :

$$
\mathbf{q}^{(c)} \approx\left(\overline{\mathbf{K}}^{(c)}-\omega^{2} \mathbf{M}^{(c)}\right)^{-1} \overline{\mathbf{K}}^{(c \rightarrow e)} \mathbf{q}_{0}^{(e)}
$$

An additional approximation consists in neglecting the elastic term of suspensions with respect to the inertia term in the dynamic behaviour of the blocked uncoupled chassis at idle $\left(\left\|\overline{\mathbf{K}}^{(c)}\right\| /\left\|\omega^{2} \mathbf{M}^{(c)}\right\| \leq\right.$ 0.05 at $25 \mathrm{~Hz}$ for the 4 cylinder engine model). While the blocked uncoupled powerplant frequency response is a unique rotation about the $T R A$ direction, the transmitted displacements on the chassis can be approximated by the expression (32).

$$
\mathbf{q}^{(c)} \approx-\left(\omega^{2} \mathbf{M}^{(c)}\right)^{-1} \overline{\mathbf{K}}^{(c \rightarrow e)} \mathbf{q}_{0}^{(e)}
$$

The inertia matrix of the chassis being generally diagonal, our approach makes it possible to obtain simple analytical relations between the displacements of the chassis and displacements of the blocked uncoupled powerplant at idle. The aim in the improvement of the vibroacoustic performances of an powerplant mounting system is the reduction in the vibratory responses of the chassis. The achievement of this objective is possible by the control of the displacements of the blocked uncoupled powerplant (32) while reducing the following coupling terms : 


$$
\left\{\begin{aligned}
u_{x}^{(c)} & =\frac{\theta_{y}^{(e)}}{\omega^{2} m(c)} \sum_{i}\left(\bar{k}_{x x i} z_{i}^{(e)}-\bar{k}_{x z i} x_{i}^{(e)}\right) \\
u_{y}^{(c)} & =\frac{\theta_{y}^{(e)}}{\omega^{2} m(c)} \sum_{i}\left(\bar{k}_{x y i} z_{i}^{(e)}-\bar{k}_{y z i} x_{i}^{(e)}\right) \\
u_{z}^{(c)} & =\frac{\theta_{y}^{(e)}}{\omega^{2} m^{(c)}} \sum_{i}\left(\bar{k}_{x z i} z_{i}^{(e)}-\bar{k}_{z z i} x_{i}^{(e)}\right) \\
\theta_{x}^{(c)} & =\frac{\theta_{y}^{(e)}}{\omega^{2} I_{x x}^{(c)}} \sum_{i}\left(-\bar{k}_{x y i} z_{i}^{(e)} z_{i}^{(c)}+\bar{k}_{y z i} x_{i}^{(e)} z_{i}^{(c)}+\bar{k}_{x z i} z_{i}^{(e)} y_{i}^{(c)}-\bar{k}_{z z i} x_{i}^{(e)} y_{i}^{(c)}\right) \\
\theta_{y}^{(c)} & =\frac{\theta_{y}^{(e)}}{\omega^{2} I_{y y}^{(c)}} \sum_{i}\left(\bar{k}_{x x i} z_{i}^{(e)} z_{i}^{(c)}-2 \bar{k}_{x z i} x_{i}^{(e)} z_{i}^{(c)}+\bar{k}_{z z i} x_{i}^{(e)} x_{i}^{(c)}\right) \\
\theta_{z}^{(c)} & =\frac{\theta_{y}^{(e)}}{\omega^{2} I_{z z}^{(c)}} \sum_{i}\left(-\bar{k}_{x x i} z_{i}^{(e)} y_{i}^{(c)}+\bar{k}_{x z i} x_{i}^{(e)} y_{i}^{(c)}+\bar{k}_{x y i} z_{i}^{(e)} x_{i}^{(c)}-\bar{k}_{y z i} x_{i}^{(e)} x_{i}^{(c)}\right)
\end{aligned}\right.
$$

The first three equations of the system (33) define the translational displacements of the chassis center of gravity. These displacements are null by applying the first three equations of the system of torque roll axis decoupling (28).

\subsection{Analytical solutions for a practical mounting systems}

To illustrate the issue of the torque roll axis strategy, we use the current mounting system for 4 cylinder front wheel drive cars. This system is typically made up of three mounts (Figure 10), two of which bear most of the weight of the powertrain while the remaining function is a roll-restrictor. The two mounts bearing the static weight are on the body rails. These are referred as Right Hand Mount (RHM) and Left Hand Mount (LHM). The roll-restrictor attaches the lower part of the powerplant to the rear cross member of the front subframe. It is referred as the Lower Torque Rod (LTR). The principal elastic axes of the resilient supporting elements are coincident with the axes of the global coordinate system $\mathcal{R}\left(k_{l m}=0\right.$ for $\left.\left.l \neq m\right)\right)$. The two mounts (RHM and LHM) are then characterized by three different stiffnesses along the three axes of the global coordinate system $\mathcal{R}$. The torque rod mainly acts in the longitudinal direction $X$, because of its low stiffness in the other directions. LTR is characterized only by a single axial stiffness $\left(\bar{k}_{x x} \neq 0\right.$ and $\left.\bar{k}_{y y}=\bar{k}_{z z}=0\right)$.

When RHM and LHM are located in the vertical plane including the $T R A$, which coincides with the plane including powerplant center of gravity and $Y$ axis of the global coordinate system $\mathcal{R}$, the coordinate distance $x^{(e)}$ of the elastic center of RHM and LHM are null (Figure 10). Moreover $\bar{k}_{z z}=0$ for LTR, so the following terms in the systems of equations (28) and (33) are null :

$$
\sum_{i} \bar{k}_{z z i} x_{i}^{(e)}=\sum_{i} \bar{k}_{z z i} x_{i}^{(e)} y_{i}^{(e)}=\sum_{i} \bar{k}_{z z i}\left(x_{i}^{(e)}\right)^{2}=\sum_{i} \bar{k}_{z z i} x_{i}^{(e)} y_{i}^{(c)}=\sum_{i} \bar{k}_{z z i} x_{i}^{(e)} x_{i}^{(c)}=0
$$


Introducing the further simplification that the $X Z$ plane and the $X Y$ plane of the global coordinate system $\mathcal{R}$ contain the centers of gravity of the powerplant and the chassis, we find that $z^{(e)}=z^{(c)}$ and $y^{(e)}=y^{(c)}$ for the three mounts (Figure 10). The aim of these simplifications is to reduce the numbers of terms in the equations and, in some instances, to reduce the number of equations that must be solved simultaneously for a complete decoupling of the TRA mode. In general, an optimization algorithm is needed to restrict the location of mounts and to ensure that the natural frequencies of the powerplant rigid-body modes lie within the desirable band while maintaining the decoupled TRA mode. The system (28) becomes :

$$
\left\{\begin{aligned}
\sum_{i}\left(\bar{k}_{x x i} z_{i}^{(e)}\right) & =0 \\
\sum_{i}\left(\bar{k}_{x x i}\left(z_{i}^{(e)}\right)^{2}\right) & =-\omega_{T R A}^{2} I_{y y}^{(e)} \\
\sum_{i}\left(-\bar{k}_{x x i} z_{i}^{(e)} y_{i}^{(e)}\right) & =0
\end{aligned}\right.
$$

Substituting the previous assumptions and the $T R A$ decoupling conditions into the equations of motion of the chassis (33), equations (36) show that the chassis is excited only in the rotational displacement about the $Y$ axis $\theta_{y}^{(c)}$. Indeed $\theta_{z}^{(c)}$ is null since the third equation of the system (35) is satisfied and $y^{(e)}=y^{(c)}$ for RHM and LHM. It is interesting to annul this coupling term because the denominator contains the lowest moment of inertia of the chassis $\left(I_{z z}^{(c)}\right)$.

$$
\left\{\begin{array}{l}
u_{c x}=u_{c y}=u_{c z}=\theta_{x}^{(c)}=0 \\
\theta_{y}^{(c)}=\frac{\theta_{0 y}^{(e)}}{\omega^{2} I_{y y}^{(c)}} \sum_{i}\left(\bar{k}_{x x i} z_{i}^{(e)} z_{i}^{(c)}\right) \\
\theta_{z}^{(c)}=\frac{\theta_{0 y}^{(e)}}{\omega^{2} I_{z z}^{(c)}} \sum_{i}\left(-\bar{k}_{x x i} z_{i}^{(e)} y_{i}^{(c)}\right)
\end{array}\right.
$$

Use of such a torque rod type mounting system increases the degrees of freedom in tuning, since the spring characteristics of the LTR do not affect in the vertical direction and the spring characteristics in the vertical direction and the roll direction are not coupled. That means that the roll mode frequency is quite easy to decrease while respecting the static requirements. Especially that the value of the coupling term between the grounded powerplant rotation and the resulting chassis rotation is also fixed by the value of the natural frequency of the powerplant roll mode. The lower will be the powerplant roll mode frequency, the lower the chassis response. 


\section{Conclusions}

This study was initiated with a desire to determine the significance of the powerplant rigid-body modes for grounded system to its in-vehicle NVH behaviour.

The current powerplant mounting strategies examined the rigid-body modes of the powertrain as it would sit on the mounts attached to the ground, i.e. neglecting the effect of the chassis. To predict correctly the issue of the traditional powerplant mounting strategies in terms of improvement of the dynamic chassis responses, it is essential to be able to analyze the phenomena induced by the coupling, and this for the whole of the excitation frequencies. The complexity of the harmonic response of the powertrain mounted on engine mounts in a vehicle cannot be understood using the traditional equations of motion. The general equations of motion are reformulated by using the coupling matrix, intrinsic with the suspended bodies and independent of the external excitation. The coupling matrix constitutes the starting point of the analysis of the traditional powerplant mounting strategies and the order of coupling enable to define their field of validity in frequency.

To format the method, we choose to analyse the torque roll axis decoupling strategy. The practical interest of this method is to force the grounded powerplant to have a frequency response only in the TRA direction with the crankshaft torque variation excitation in all frequency range. There is only one pure mode excited and then only one frequency to move away from the excitation frequency to reduce powerplant vibration. This is still right in the full vehicle model only if the interactions between the powerplant and the chassis are negligible, i.e. at frequencies where the eigenvalues of coupling are low. The use of a simple rigid-body representation of the powerplant and the chassis enable to concentrate only on the physique of the coupling problem. It is obvious that such a simplicity occults the effects of chassis flexibility or the modal properties of the cradle on the dynamic response. Future work should include the experimental verification of the simulation through measurements of the motion of the powerplant mounting system.

For road/wheel inputs, a different model should be considered. In this case it is necessary to reduce the acceleration due to the engine shake. The engine shake is due to a dynamic interaction between the vehicle suspensions and the powerplant mounting system. For future enhancements to this study, investigation will be conducted to analyze the order of coupling between the wheel suspension system and the powerplant mounting system. 


\section{References}

[Bessac F. and Guyader, 1996] Bessac F., G. L. and Guyader, J.-L. (1996). Coupling eigenvalues and eigenvectors : A tool for investigating the vibroacoustic behaviour of coupled vibrating systems. Journal of Sound and Vibration., 25(5):881-899.

[Brach, 1997] Brach, R. (1997). Automotive powerplant isolation strategies. SAE Technical Paper Series, 971942.

[Gray et al., 1990] Gray, S., Arai, T., and Kubozuka, T. (1990). Development of an engine mount optimization method using modal parameters. SAE Technicla Paper Series, 932898.

[Hadi and Sachdeva, 2003] Hadi, R. and Sachdeva, D. (2003). Effect of mounting strategy on vehicle NVH. SAE Technical Paper, 2003-01-1467.

[Julien, 1960] Julien, M. (1960). Dynamique de la Voiture Automobile. Editions Technip, 27 rue Ginoux 75737 Paris Cedex 15.

[Kano and Hayashi, 1994] Kano, S. and Hayashi, S. (1994). Optimization method for mount layout and mount specifications of vehicule sub-frame. JSAE Technical Paper Series, 940077.

[Khajepour and Geisberger, 2002] Khajepour, A. and Geisberger, A. (2002). Engine mounting systems from passive to active. Recent Research Development Sound and Vibration., 81-7895-0316:697-718.

[Patton and Geck, 1984] Patton, R. and Geck, P. (1984). Front wheel drive engine mount optimization. SAE Technical Paper Series, 840736.

[Saitoh and Igarashi, 1990] Saitoh, S. and Igarashi, M. (1990). Optimization study of engine mounting systems. Automobile testing division Suzuki motor company, JAPAN.

[Shane Sui and Hoppe, 2003] Shane Sui, J. and Hoppe, L. (2003). Powertrain mounting design principles to achieve optimum vibration isolation with demonstration tools. SAE Technical Papers, 2003-01-1476.

[Singh and Jeong, 2000] Singh, R. and Jeong, T. (2000). Analytical methods of decoupling the automotive engine torque roll axis. Journal Sound and Vibration, 234:85-114.

[Sirafi and Qatu, 2003] Sirafi, M. and Qatu, M. (2003). Accurate modeling for the powertrain and subframe modes. SAE technical papers, 2003-01-1469. 


\begin{tabular}{|c|c|c|c|c|c|c|c|c|c|c|}
\hline \multirow[t]{2}{*}{ Engine } & \multirow[t]{2}{*}{ Mount \# } & \multicolumn{3}{|c|}{$\begin{array}{l}\text { Location } \\
\qquad(m m)\end{array}$} & \multicolumn{3}{|c|}{$\begin{array}{c}\text { Orientation } \\
\text { Euler angles }(\mathrm{deg})\end{array}$} & \multicolumn{3}{|c|}{$\begin{array}{l}\text { Stiffness } \\
(N / m m)\end{array}$} \\
\hline & & $X$ & $Y$ & $Z$ & $\Phi$ & $\Theta$ & $\Psi$ & $k_{u}$ & $k_{v}$ & $k_{w}$ \\
\hline \multirow{3}{*}{ 3-cylinder } & 1 & -330 & 210 & 270 & 0 & -90 & -67 & 65 & 40 & 40 \\
\hline & 2 & 90 & 200 & 250 & 0 & -90 & -112 & 65 & 40 & 40 \\
\hline & 3 & -100 & -230 & 95 & 0 & -90 & -90 & 140 & 60 & 60 \\
\hline \multirow{3}{*}{ 4-cylinder } & 1 & -184 & -390 & 299 & 0 & -90 & -90 & 216 & 180 & 60 \\
\hline & 2 & -242 & 511 & 493 & 0 & -90 & -90 & 144 & 132 & 30 \\
\hline & 3 & 19 & -53 & 35 & 90 & 10 & -90 & 120 & 0 & 0 \\
\hline \multirow{4}{*}{ 6-cylinder } & 1 & -485 & 11 & 133 & 0 & -90 & -67 & 200 & 70 & 70 \\
\hline & 2 & 85 & 11 & 152 & 0 & -90 & -112 & 200 & 70 & 70 \\
\hline & 3 & -217 & 482 & 10 & 0 & -90 & -90 & 400 & 135 & 135 \\
\hline & 4 & -194 & -432 & -24 & 0 & -90 & -90 & 400 & 135 & 135 \\
\hline
\end{tabular}

Table 1: Mounting system characteristics of 3 models of front wheel drive cars (a 3, 4 and 6 cylinder engine) 


\begin{tabular}{|c|cc|c|c|}
\cline { 3 - 5 } \multicolumn{2}{c|}{} & 3-cylinder & 4-cylinder & 6-cylinder \\
\hline \multirow{4}{*}{ Mass $(k g)$} & 135 & 173 & 342 \\
\hline \multirow{4}{*}{ Center of mass $(m m)$} & $Y$ & -222 & -222 & -194 \\
\cline { 3 - 5 } & $Y$ & 103 & 103 & 16 \\
\cline { 2 - 5 } & $Z$ & 292 & 292 & 200 \\
\hline \multirow{4}{*}{ Moment of inertia $\left(k g . m^{2}\right)$} & $I_{x x}$ & 5.15 & 10.37 & 28.03 \\
\cline { 2 - 5 } & $I_{y y}$ & 2.85 & 5.53 & 17.1 \\
\cline { 2 - 5 } & $I_{z z}$ & 4.44 & 9.77 & 26.77 \\
\cline { 2 - 5 } & $I_{x y}$ & -0.47 & -1.72 & -1.66 \\
\cline { 2 - 5 } & $I_{x z}$ & -0.52 & -0.07 & -0.03 \\
\cline { 2 - 5 } & $I_{y z}$ & 0.43 & 1.84 & 3.97 \\
\hline
\end{tabular}

Table 2: Powerplant mass characteristics of 3 models of front wheel drive cars (a 3, 4 and 6 cylinder engine).

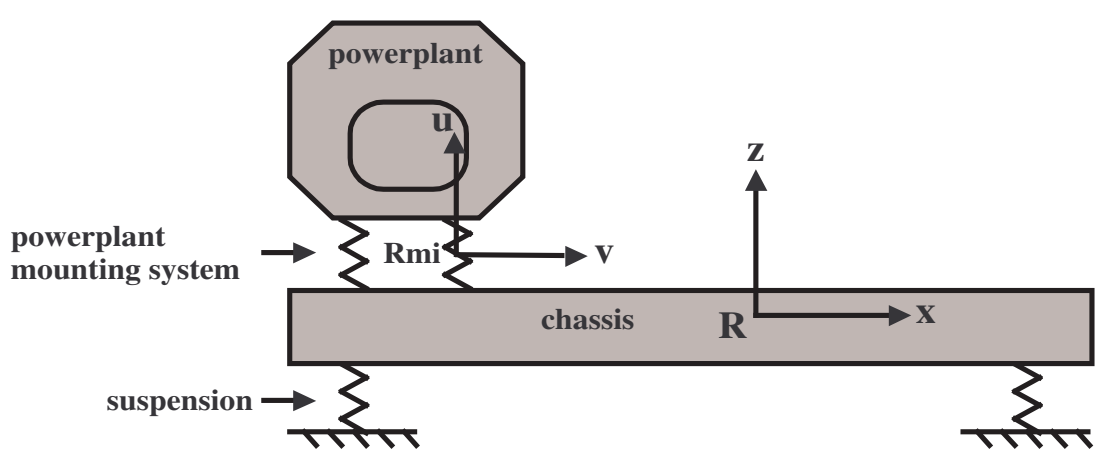

Figure 1: Powerplant mounting model 


\begin{tabular}{|c|cccccc|}
\hline Powerplant frequencies $(\mathrm{Hz})$ & 1 st & 2nd & 3rd & 4 th & 5 th & 6 th \\
\hline $\begin{array}{c}3 \text { cylinder engine } \\
\text { 4 cylinder engine }\end{array}$ & 4.12 & 4.92 & 6.55 & 9.14 & 10.73 & 14.89 \\
6 cylinder engine & 3.28 & 5.99 & 6.57 & 8.80 & 12.58 & 12.67 \\
\hline Chassis frequencies $(\mathrm{Hz})$ & 1 st & 2 nd & 3 rd & 4 th & 5 th & 6 th \\
\hline $\begin{array}{c}3 \text { cylinder engine } \\
4 \text { cylinder engine }\end{array}$ & 3.48 & 4.89 & 5.01 & 5.32 & 5.62 & 7.07 \\
6 cylinder engine & 3.72 & 4.72 & 5.05 & 5.58 & 5.76 & 6.64 \\
\hline
\end{tabular}

Table 3: Frequencies of the rigid body modes of the blocked uncoupled powerplant and chassis

\begin{tabular}{|l|cccccccccccc|}
\hline Frequencies $(\mathrm{Hz})$ & 1 st & 2nd & 3rd & 4 th & 5 th & 6 th & 7 th & 8 th & 9 th & 10th & 11 th & 12 th \\
\hline 3 cylinder engine & 3.01 & 3.78 & 4.39 & 4.84 & 5.98 & 6.11 & 6.34 & 6.55 & 8.09 & 9.54 & 11.41 & 15.34 \\
4 cylinder engine & 2.58 & 3.39 & 3.78 & 4.01 & 4.88 & 5.02 & 5.43 & 7.15 & 7.97 & 9.28 & 12.67 & 12.95 \\
6 cylinder engine & 2.28 & 2.67 & 3.02 & 3.39 & 4.07 & 4.14 & 5.31 & 6.68 & 7.85 & 9.35 & 11.93 & 13.28 \\
\hline
\end{tabular}

Table 4: Frequencies of the rigid body modes of the powerplant on mounting system

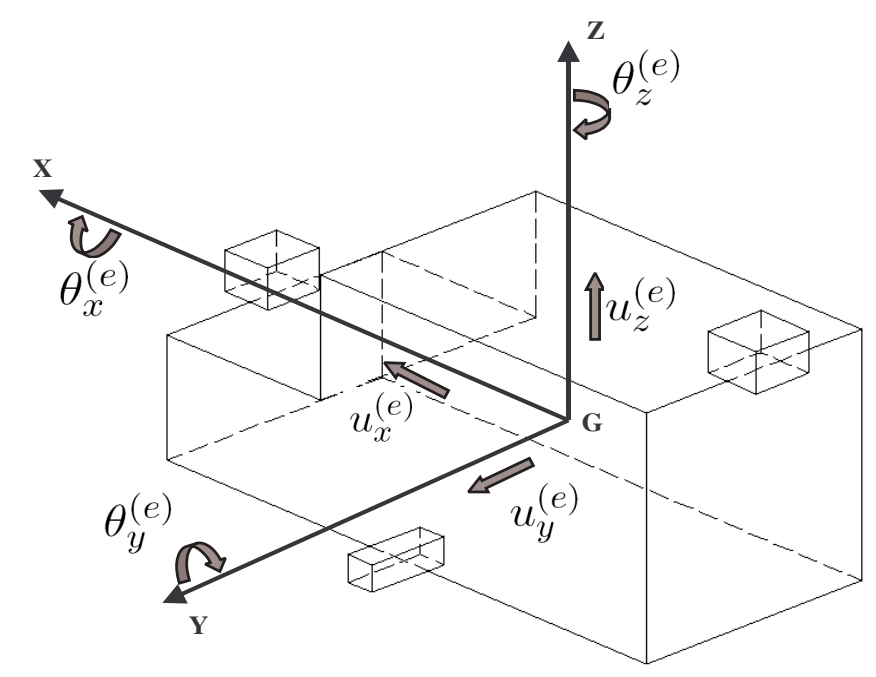

Figure 2: Translational $\mathbf{u}$ and rotational $\boldsymbol{\theta}$ displacements of the powerplant center of gravity 


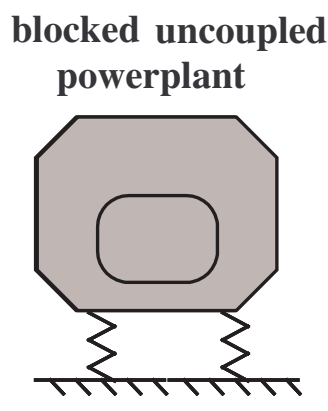

(a)

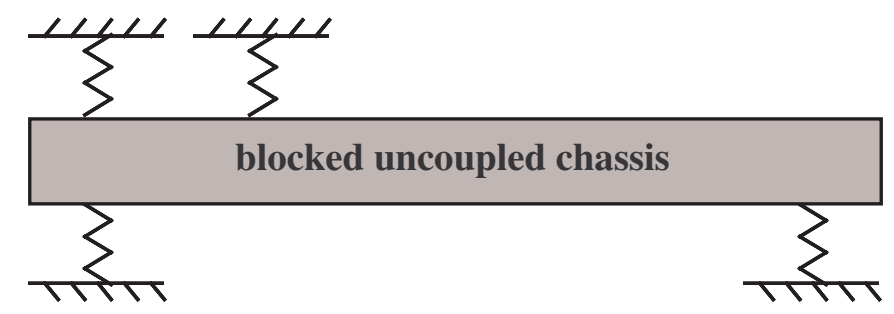

(b)

Figure 3: Blocked uncoupled bodies

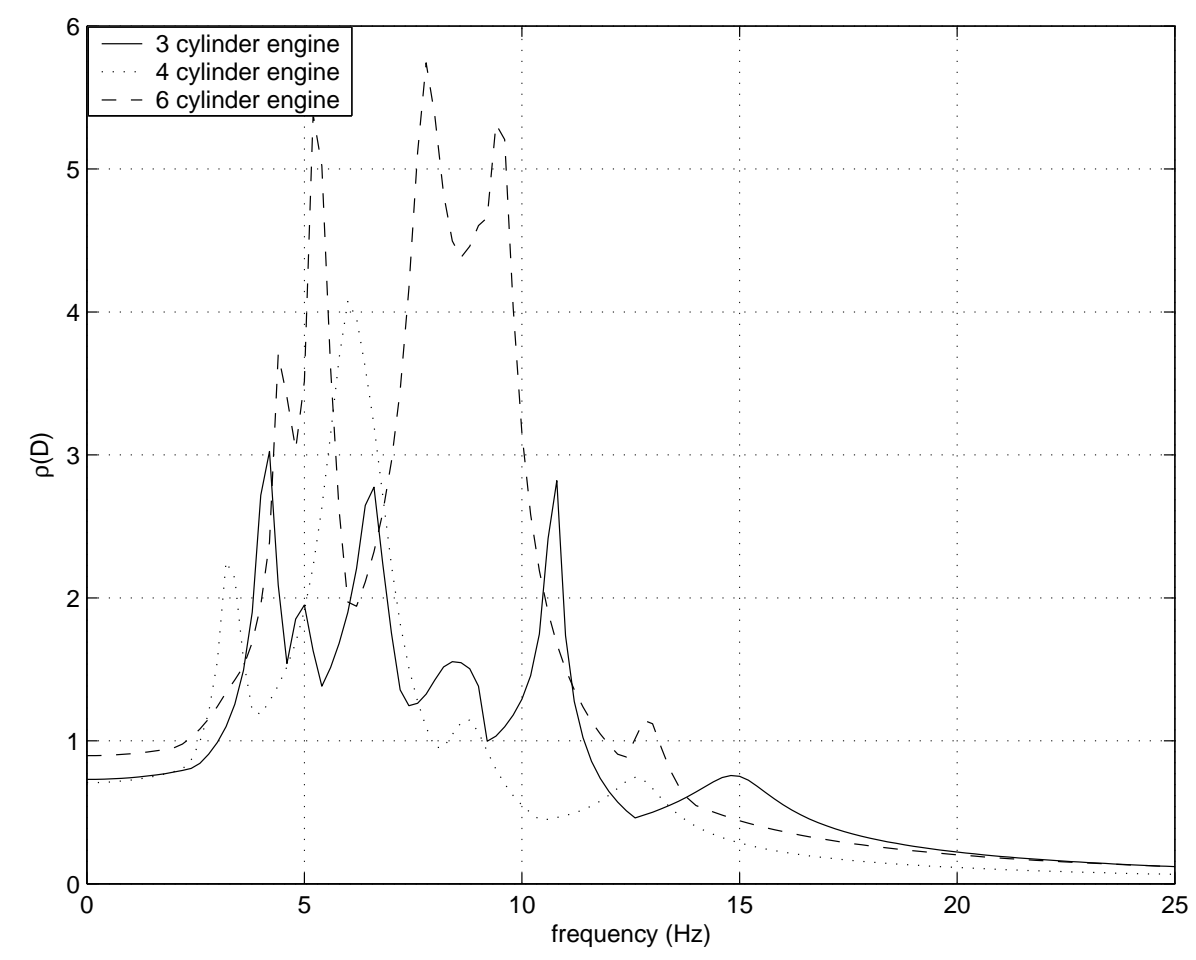

Figure 4: Spectral radius of the coupling matrix $\mathbf{D}$ of 3 models of front wheel drive cars (a 3,4 and 6 cylinder engine) 


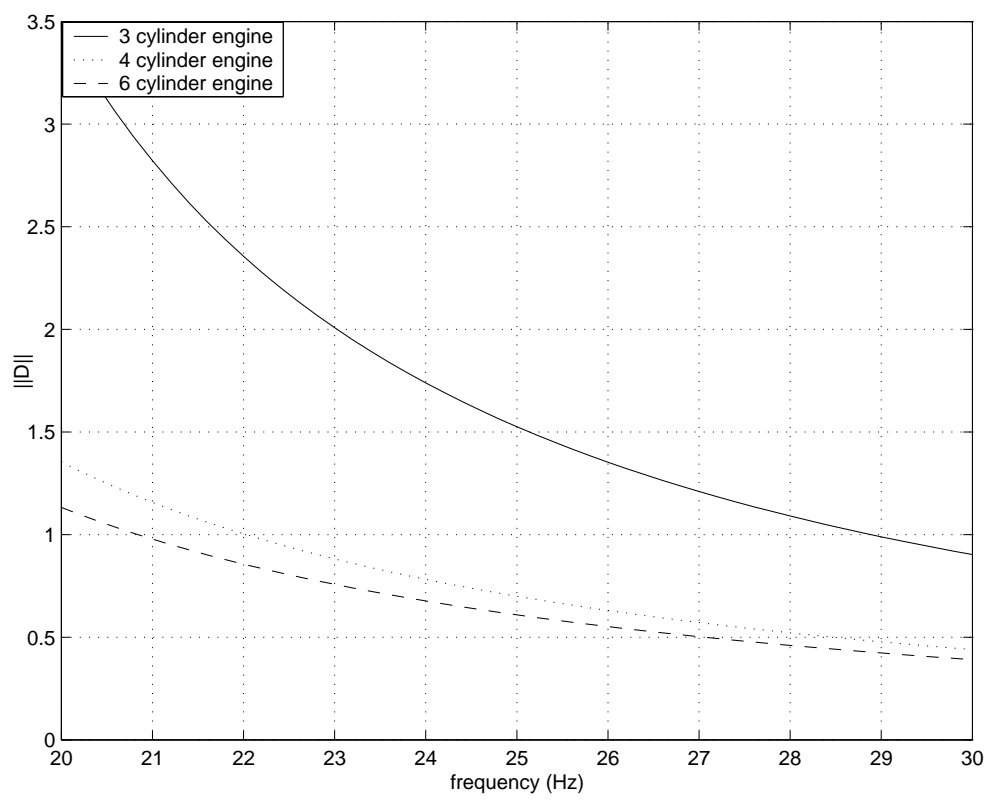

Figure 5: Frobenius norm of the coupling matrix $\mathbf{D}$ of 3 models of front wheel drive cars (a 3, 4 and 6 cylinder engine)
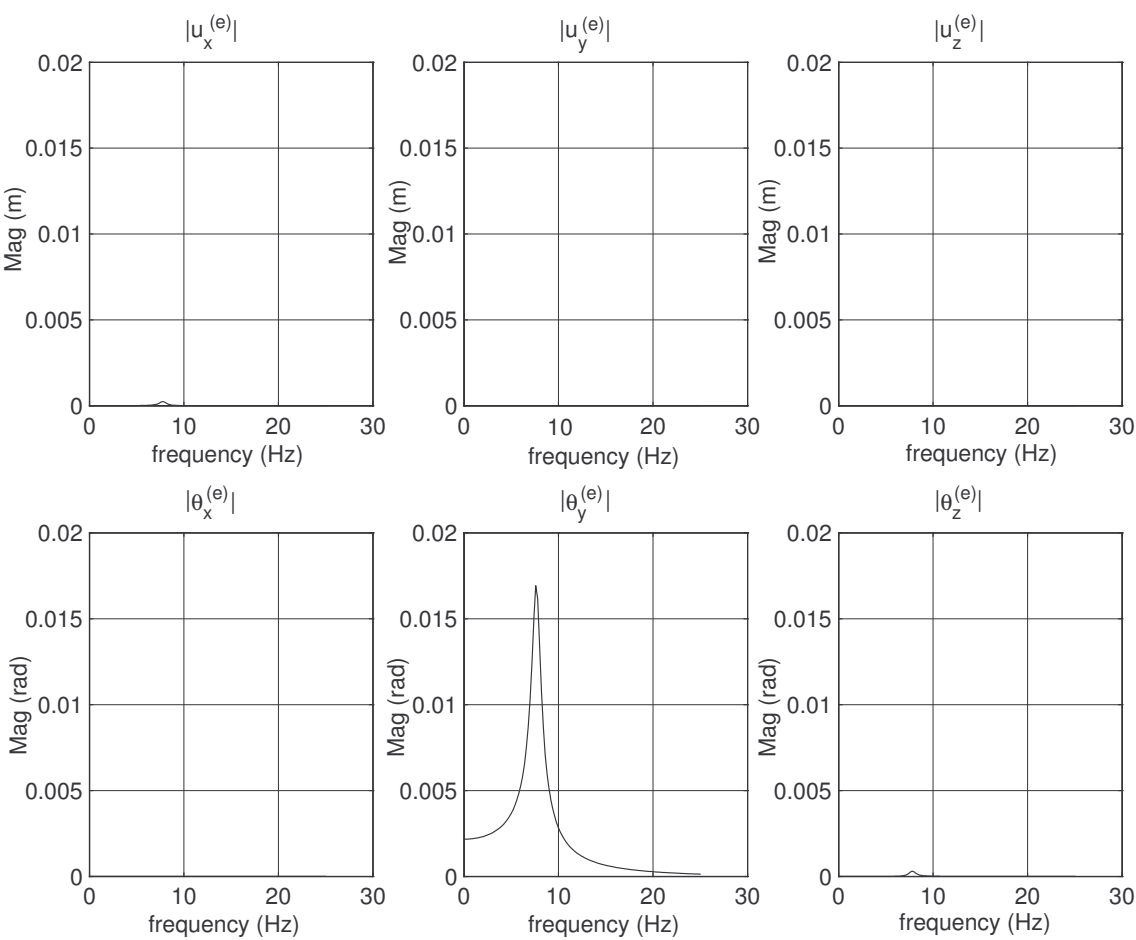

Figure 6: Decoupled frequency response of the powerplant center of gravity of the 4 cylinder engine model in the global coordinate system $\mathcal{R}$ with only crankshaft torque excitation 

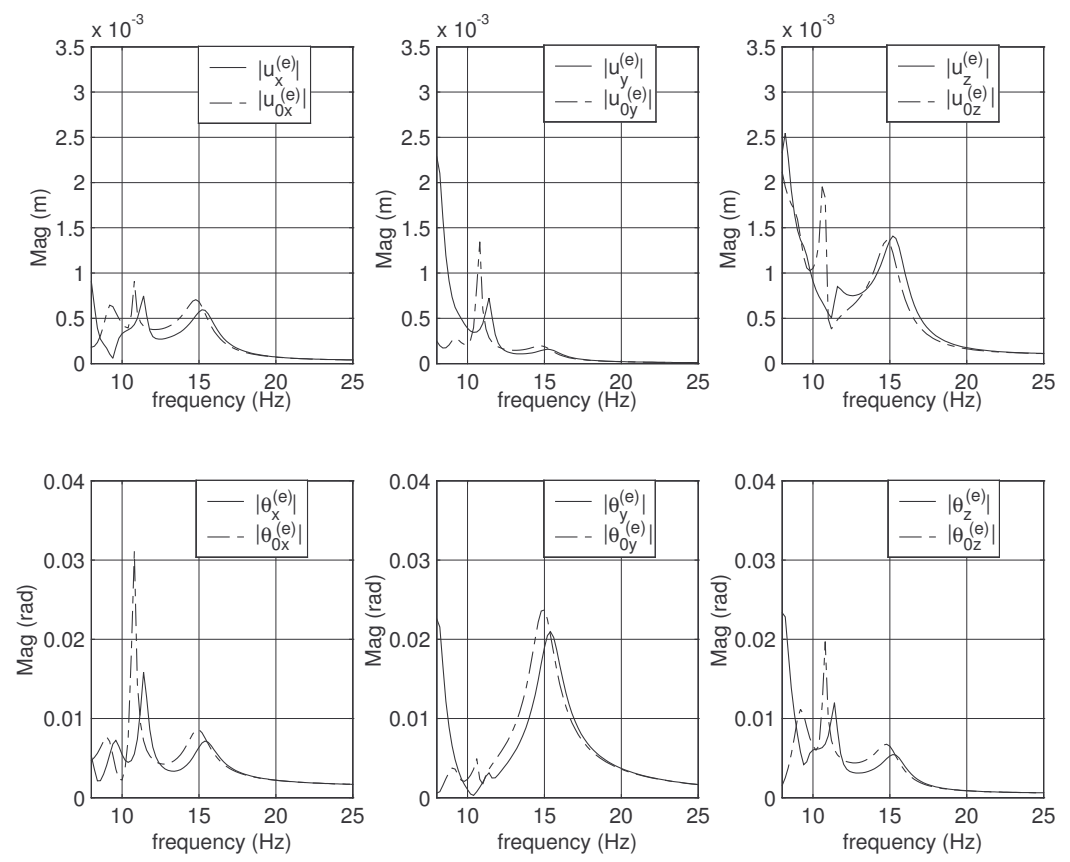

Figure 7: Frequency response of the powerplant center of gravity of the 3 cylinder engine model in the global coordinate system $\mathcal{R}$ for idle excitation
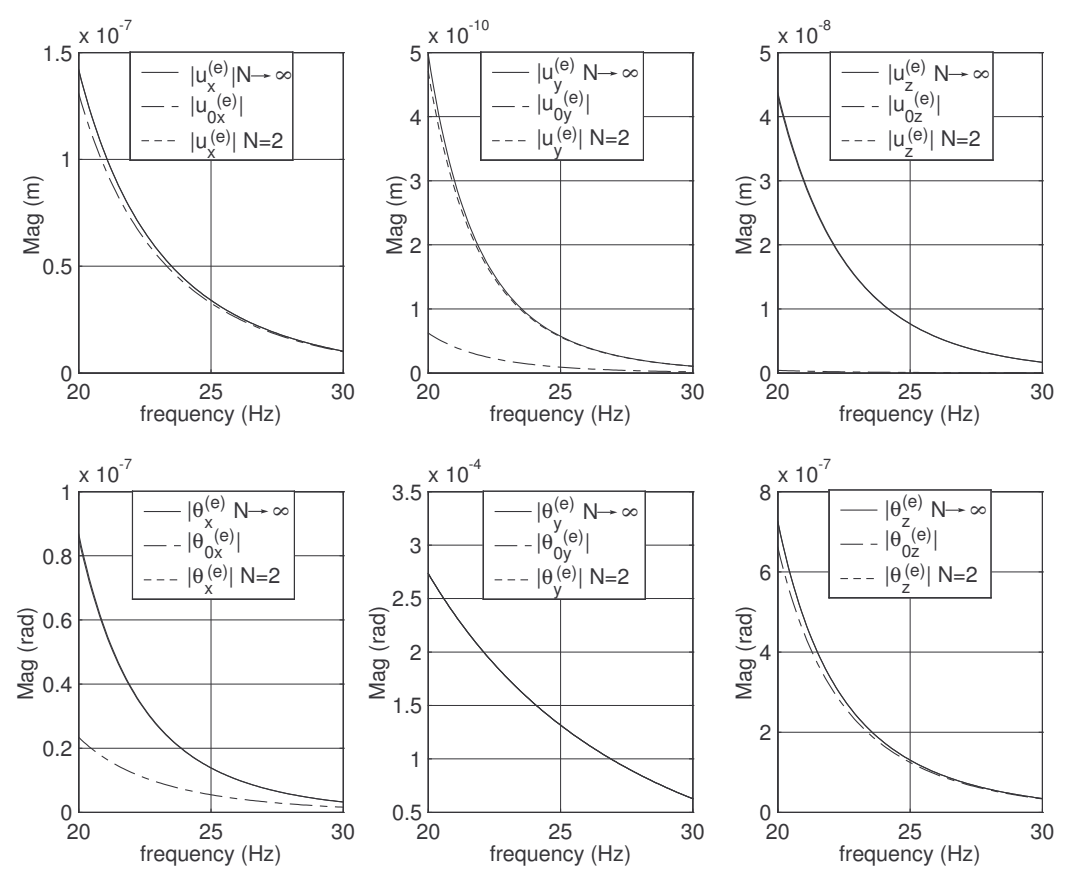

Figure 8: Decoupled frequency response of the powerplant center of gravity of the 4 cylinder engine model in the global coordinate system $\mathcal{R}$ with only crankshaft torque excitation 

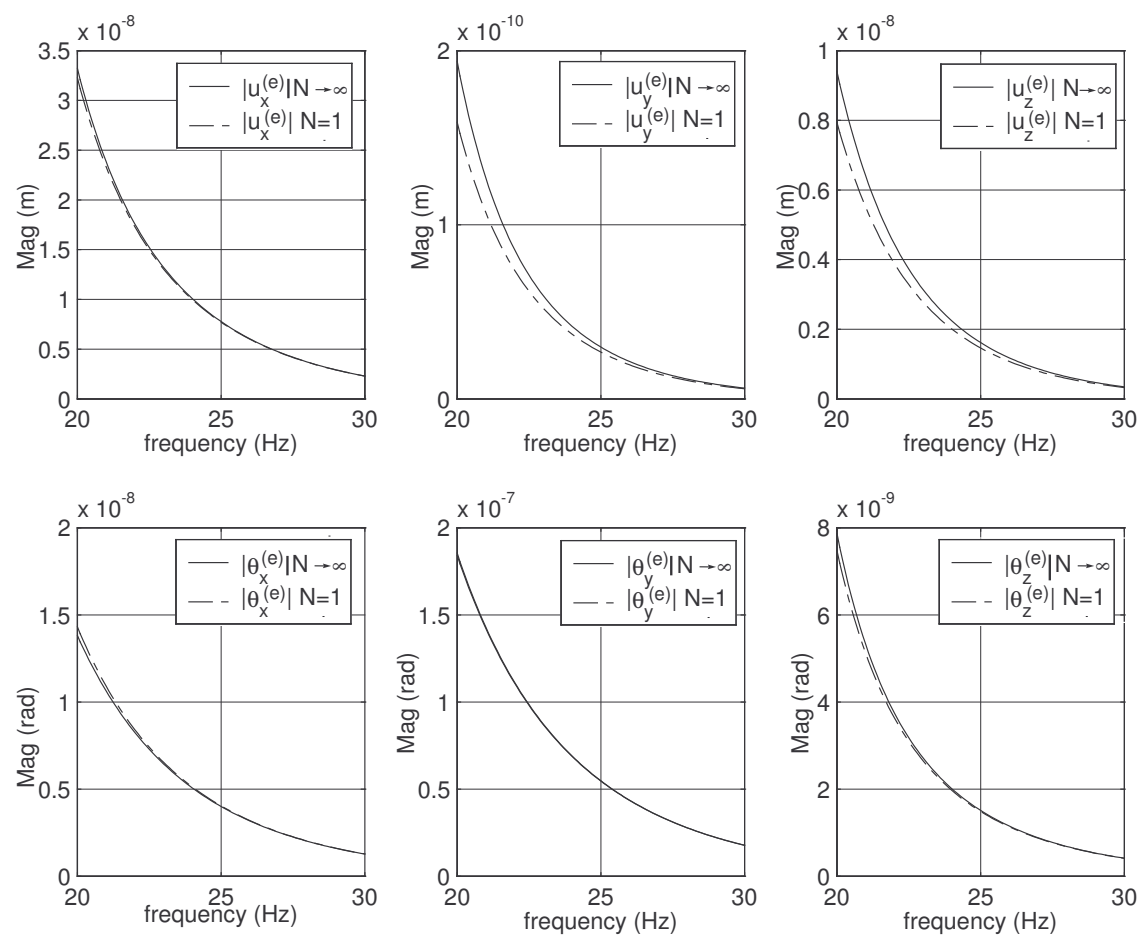

Figure 9: Decoupled frequency response the chassis center of gravity of the 4 cylinder engine model in the global coordinate system $\mathcal{R}$ with only crankshaft torque excitation
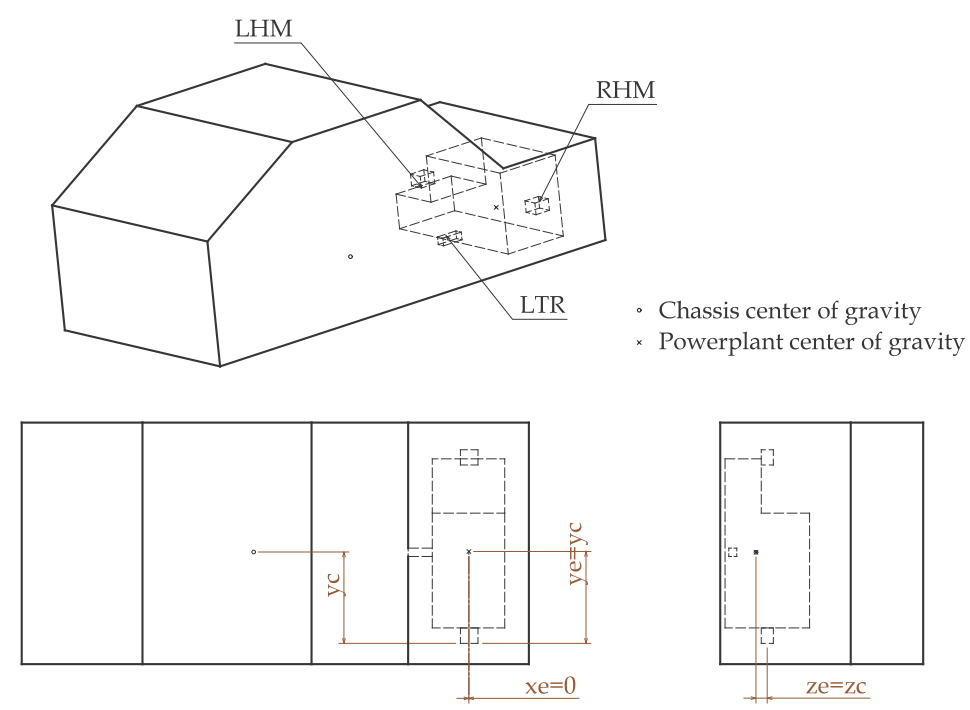

Figure 10: Powerplant mounting model and layout of the three engine mounts 